\title{
Effect of Modulated Signals on Vibrational Resonance in a Harmonically Trapped Potential System
}

\author{
B. Bhuvaneshwari ${ }^{1}$, S. V. Priyatharsini ${ }^{1}$, V. Chinnathambi ${ }^{1 *}$, S. Rajasekar ${ }^{2}$ \\ ${ }^{1}$ Department of Physics, Sadakathullah Appa College, Tirunelveli-627 011, Tamilnadu, India \\ ${ }^{2}$ School of Physics, Bharathidasan University, Tiruchirapalli 620 024, Tamilnadu, India
}

Received 7 March 2021, accepted in final revised form 17 May 2021

\begin{abstract}
We consider a harmonically trapped potential system driven by modulated signals with two widely different frequencies $\omega$ and $\Omega$, where $\Omega>>\omega$. The forms of modulated signals are amplitude modulated (AM) and frequency-modulated (FM) signals. An amplitudemodulated external signal is consisting of a low-frequency $(\omega)$ component and two highfrequencies $(\Omega+\omega)$ and $(\Omega-\omega)$ whereas the frequency modulated signal consisting of the frequency components such as $f \sin \omega t \cos (g \cos \Omega t)$ and $f \sin (g \cos \Omega t) \cos \omega t$. Depending upon the values of the parameters in the potential function, an odd number of potential wells of different depths can be generated. We numerically investigate the effect of these modulated signals on vibrational resonance (VR) in single-well, three-well, five-well and seven-well potentials. Different from traditional VR theory in the present paper, the enhancement of VR is made by the amplitudes of the AM and FM signals. We show the enhanced response amplitude $(Q)$ at the low-frequency $\omega$, showing the greater number of resonance peaks and non-decay response amplitude on the response amplitude curve due to the modulated signals in all the potential wells. Furthermore, the response amplitude of the system driven by the AM signal exhibits hysteresis and a jump phenomenon. Such behavior of $Q$ is not observed in the system driven by the FM signal.
\end{abstract}

Keywords: Vibrational resonance; Modulated signals; Harmonically trapped potential; Response amplitude.

(C) 2021 JSR Publications. ISSN: 2070-0237 (Print); 2070-0245 (Online). All rights reserved. doi: http://dx.doi.org/10.3329/jsr.v13i3.52318 J. Sci. Res. 13 (3), 797-807 (2021)

\section{Introduction}

Nonlinear systems are capable of displaying a variety of regular and irregular dynamics. Resonance is one of the fundamental phenomena exhibited by linear and nonlinear systems. In a dynamical system driven by an external periodic force, when the frequency of the force is varied, in a typical case, the amplitude of the oscillation decreases and reaches a significantly large value of a frequency and then decreases. The realization of maximum amplitude is called resonance. The effect of resonance is to produce a large amplitude oscillation. Resonance occurs in many branches of physics, engineering and biology.

\footnotetext{
*Corresponding author: veerchinnathambi@ gmail.com
} 
Various types of resonance can be realized in nonlinear systems such as the stochastic resonance [1,2], coherence resonance [3,4], auto resonance [5,6], ghost resonance [7-9], chaos resonance [10,11], parametric resonance [12,13] and vibrational resonance [14-18]. The present paper is concerned with vibrational resonance. The phenomenon of vibrational resonance (VR) in which the system responds to a weak periodic signal can be enhanced by applying the high-frequency periodic perturbation of appropriate amplitude. The analysis of VR has received considerable interest in recent years because of its importance in a wide variety of contexts in science and engineering. This phenomenon was originally discovered by Landa and McClintok [14] in a bistable system. More recently, Oyeleke et al. [12] studied the occurrence of VR in the gyroscope model driven by dual-frequency forces such as a parametric excitation and an additive periodic force. In addition to the well-known method of tuning the strength of the high-frequency field in VR, they have shown that the occurrence of VR in this model when the low-frequency parametric excitation is tuned. Furthermore, they obtained a higher response amplitude and a wider response bandwidth when a low-frequency parametric excitation force cooperates with a high-frequency additive forcing. Due to wide interest in the multifrequency signals, VR has been investigated in many systems, such as bistable system [19-22], monostable system [23], asymmetric system [24], spatially periodic potential system [25,26], time-delayed system [27] and many more.

Generally, in the VR studies, a nonlinear system driven by a weak periodic force, say, $f \sin \omega t$ and a high-frequency force $g \sin \Omega t$ with $\Omega>>\omega$, that is, the total external force is $F(t)=f \sin \omega t+g \sin \Omega t$. System response for different kinds of external force is an interesting topic and the article presented a non-conventional type of VR. The prime aim of the present paper is to analyze VR first by an amplitude-modulated force $F_{A M}(t)=(f+$ $2 g \cos \Omega t) \sin \omega t$ which can also be written as $F_{A M}(t)=f \sin \omega t+g \cos (\Omega+\omega) t+g$ $\cos (\Omega-\omega) t$. When $\Omega>>\omega$, the amplitude modulated force $F_{A M}(t)$ can also be treated as consisting of a low-frequency force $f \sin \omega t$ and two high-frequency force with frequencies $(\Omega+\omega)$ and $(\Omega-\omega)$. Then we analyze VR by the frequency-modulated force $F_{F M}(t)=f \sin (\omega t+g \cos \Omega t)$ which can also be written as $F_{F M}(t)=f \sin \omega t \cos (g \cos \Omega t)$ $+f \sin (g \cos \Omega t) \cos \omega t$. Certain notable earlier studies on nonlinear systems are subjected to modulated signals with and without the restriction of $\Omega \gg \omega[18,28-32]$. There are some interesting VR features in the harmonically trapped system driven by modulated signals $\left(\mathrm{F}_{\mathrm{AM}}(\mathrm{t})\right.$ or $\left.\mathrm{F}_{\mathrm{FM}}(\mathrm{t})\right)$ compared to the case when it is driven by the force $F_{l}(t)=f \sin$ $\omega t+g \sin \Omega t$. When the system is subjected to the force $F_{l}(t)$, the response amplitude exhibits multiple peaks, the value at the resonance of the response amplitude $Q(\omega)=$ $1 /(d \omega)$, and approaches a non-zero limiting value when the amplitude of the highfrequency signals is varied. Interestingly, in contrast to this result, when the system is subjected with the external force $F_{A M}(t)$ or $F_{F M}(t)$, multiple resonance peaks occur, where $Q(\omega) \gg 1 /(\mathrm{d} \omega)$ at some resonance peaks and $Q(\omega)$ does not decay to zero even for very large values of $\mathrm{g}$. Further, the response amplitude curve displays hysteresis and a jump phenomenon. These are the main results of this research work. 
The outline of the paper is as follows. In Section 2, we present the equation of motion of a harmonically trapped potential system with modulated signals. We study the effect of the parameter $\beta$ on the shape of the potential and the number of equilibrium points. In Section 3, we numerically analyze the effect of modulated signals on VR in harmonically trapped potential system. First, we analyze the effect of AM signal and then FM signal on vibrational resonance. We observe single and multiple resonances with the variation of the control parameters $g$ and $\Omega$ in all the signals. The conclusion is drawn in Section 4.

\section{Harmonically Trapped Potential System with Modulated Signals}

The equation of motion of a harmonically trapped potential system with Amplitude Modulated (AM) signal is given by

$$
\ddot{x}+d \dot{x}+\omega_{0}^{2}+\beta \sin x=(f+2 g \cos \Omega t) \sin \omega t,
$$

with the use of the formula $2 \sin \omega t \cos \Omega t=\sin (\omega+\Omega) t+\sin (\Omega-\omega) t$, Eq.(1) takes the form

$$
\ddot{x}+d \dot{x}+\omega_{0}^{2}+\beta \sin x=f \sin \omega t+g \sin (\omega+\Omega) t+g \sin (\Omega-\omega) t,
$$

and Frequency Modulated (FM) signal is given by

$$
\ddot{x}+d \dot{x}+\omega_{0}^{2}+\beta \sin x=f \sin (\omega t+g \cos \Omega t),
$$

with the expansion of $\sin (\omega t+g \cos \Omega t)=\sin \omega t \cos (g \cos \Omega \mathrm{t})+\sin (\mathrm{g} \cos \Omega \mathrm{t}) \cos \omega t$, Eq.(3) takes the form

$$
\ddot{x}+d \dot{x}+\omega_{0}^{2}+\beta \sin x=f \sin \omega t \cos (g \cos \Omega t)+f \sin (g \cos \Omega t) \cos \omega t,
$$

Where $\omega_{0}{ }^{2}$ is the natural frequency, $d>0$ is the damping parameter of the system and $\beta$ is the constant parameter which plays the role of nonlinear parameter, $f$ and $g$ are the amplitudes of the signals, $\omega$ and $\Omega$ are the two frequencies of the signals with $\Omega>>\omega$. We call the systems with the amplitude modulated signal (Eq. 2) and frequency modulated signal (Eq. 4) as system-1 and system-2, respectively. Recently, many researchers studied the occurrence of vibrational resonance in a harmonically trapped potential system. Particularly, Abirami et al. [33] investigated the role of the shape of potentials on vibrational resonance, and Yang et al. [34] studied the effect of linear time-delay on vibrational resonance in a harmonically trapped potential system driven by a biharmonic force with two widely different frequencies $\omega$ and $\Omega$ with $\omega<<\Omega$. In the present work, we are interested in studying numerically the effect of modulated signal on vibrational resonance in a harmonically trapped potential system with two widely different frequencies $\omega$ and $\Omega$ with $\omega<<\Omega$.

The harmonically trapped potential satisfies the following form in the absence of damping is,

$$
V(x)=\frac{1}{2} \omega_{0}^{2} x^{2}-\beta \cos x, \quad \omega_{0}^{2}, \beta>0
$$

Depending upon the parameters $\omega_{0}{ }^{2}$ and $\beta$, we can generate an odd number of potential wells of different depths. Fig. 1 shows the shapes of the potential for four fixed values of $\beta$ with $\omega_{0}{ }^{2}=1$. From Fig. 1 we can clearly observe, the number of potential wells changes from one to three, three to five, and five to seven with the increase of $\beta$ values from 3 to 5 , 
5 to 15 , and 15 to 20 . Further an increase of $\beta$, we can generate an odd number of potential wells. The equation for the root of the trapped potential system is

$$
\omega_{0}^{2} x^{*}+\beta \sin x^{*}=0
$$

$X^{*}=0$ is always the root of the equation (6). Since the potential is symmetrical about $x=$ $0, x^{*}$ is also a root equation (6). It is not easy to find an analytical expression for $x^{*}$ from Eq. (6). However, we can determine all the roots of Eq. (6) numerically by employing Newton-Raphson method. Depending on the values of $\beta$ the system has $4 n-3, n=1,2$, $3 \ldots$, number of equilibrium points while $V(x)$ has $(2 n-1), n=1,2,3 \ldots$, potential wells. At $\beta=(4 n-1) \pi, n=1,2,3, \ldots$, the number of potential wells and equilibrium points change will occur.
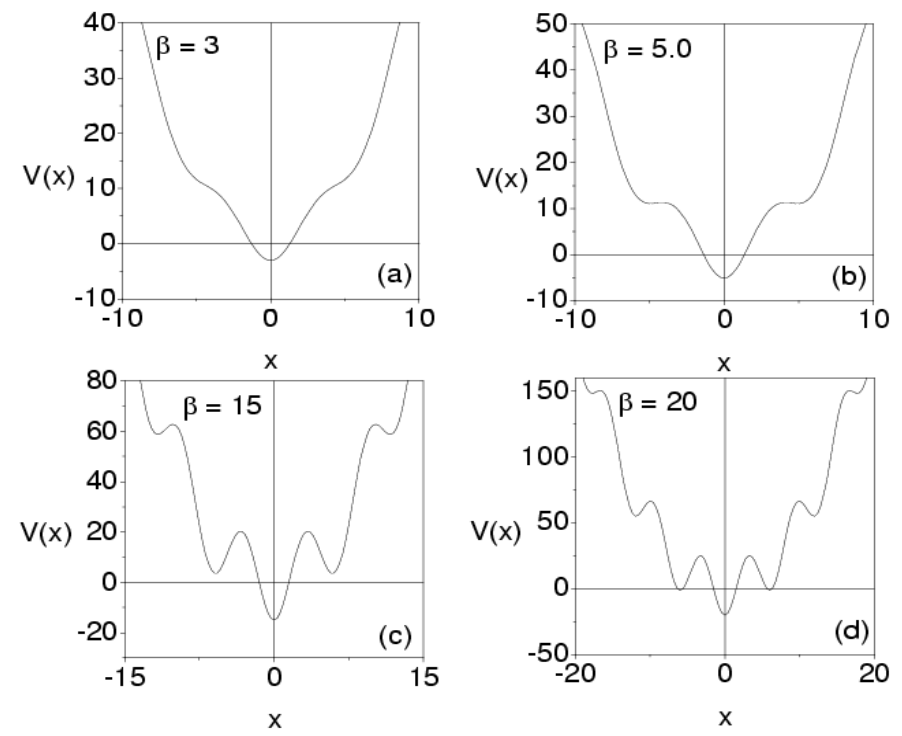

Fig. 1. The shape of the potential (Eq. 5) with $\omega_{0}^{2}=1$ for four values of $\beta$.

\section{Effect of Modulated Signals on VR in a Harmonically Trapped Potential System}

In the present work, we study the effect of modulated signals on VR in system-1 and system-2 numerically. To compute the response amplitude $(Q)$ of the slow component $X$ of $x(t)$, we numerically integrate the systems (1) and (2) using fourth-order Runge- Kutta method with time step size $(2 \pi / \omega) / 1000$. The first $10^{3}$ drive cycles are left as transient and the value of $x(t)$ corresponding to the next 500 drive cycles are used to compute the response amplitude $(Q)$. From the numerical solution of $x(t)$, the response amplitude $(Q)$ is computed from the equations,

$$
\begin{aligned}
& Q_{s}=\frac{2}{n T} \int_{0}^{n T} x(t) \sin \omega t d t, \\
& Q_{c}=\frac{2}{n T} \int_{0}^{n T} x(t) \cos \omega t d t,
\end{aligned}
$$


where $T=2 \pi / \omega$ is the period of the response and $n$ is taken as 500. Then $Q=$ $\sqrt{Q_{S}^{2}+Q_{c}^{2}}$. In all the systems (1) and (2), we fix the values of the parameters as $\omega_{0}^{2}=1.0$, $d=0.5, \omega=0.1, \Omega=1.0,5.0$ and $\beta=3,5,15$ and 20 .

\subsection{Analysis of response amplitude $Q$ with an $A M$ signal}

In this section, we numerically analyze the occurrence of VR with an AM signal for a parameter value of $\beta$ and treat the amplitude $(g)$ and frequency $(\Omega)$ of the high-frequency signal as the control parameters. We analyze the effect of AM signal for two frequencies ratio, namely, $\Omega / \omega=10$ and $\Omega / \omega=50$ with $f=0.1$ and $\omega=0.1$. First, we study the occurrence of VR for the frequency ratio $\Omega / \omega=10$. For this frequency ratio, the evolution of the response amplitude Q versus the signal amplitude $\mathrm{g}$ is shown in Fig. 2 for $f=0.1, \omega$ $=0.1$, and four values of $\beta$, such as $\beta=3,5,15,20$. In Fig. 2, for $\beta=3$ (single-well potential (Fig. 1a)) and $\beta=5$ (three-well potential (Fig. 1b)), no resonances occur when $g$ $<1.6005$ (solid line) and $g<2.619$ (dashed curve) and multiple resonances occur when $g$ $>1.6005$ and $g>2.619$. But for $\beta=15$ (five-well potential (Fig. 1c)) and $\beta=20$ (sevenwell potential (Fig. 1d)), no resonances occur in the interval $0<g<7.7227$ and $0<g<$ 10.2924 and multiple resonances occur when $g>7.7227$ and 10.2924 which are represented by dotted and dashed dot curves in Fig. 2 . For the frequency ratio $\Omega / \omega=10$, it is clearly noticed that as $\beta$ (number of potential wells) increases, the non-resonant region also increases, which is evident in Fig. 2. Next, we study the occurrence of VR for the frequency ratio $\Omega / \omega=50$. The corresponding numerical results are shown in Fig. 3. Fig. 3 shows the variation of numerically computed $\mathrm{Q}$ against the control parameter $\mathrm{g}$ for the frequency ratio $\Omega / \omega=50$ with $\omega=0.1, f=0.1$ and $\beta=3,5,15,20$. In Figs. 3(a) and 3(b) for $\beta=3$ and $\beta=5, g$ linearly increases with $\mathrm{Q}$, and no resonance takes place. For $\beta=15$, no resonances occur in the interval $0<g<5: 7986$ and further increase of $\mathrm{g}$ multiple resonances are observed when $g>5.7986$. For $\beta=20$, resonance is not observed in the interval $0<g<2.3651$ and multiple resonances occur when $g>2.3651$. Due to the effect of AM signal, for the frequency ratio $\Omega / \omega=10$, VR is observed for all the potential well but for the frequency ratio $\Omega / \omega=50$, VR is not observed in single-well and three-well potentials, and it is observed in five-well and seven-well potentials. Further $Q(\omega)$ is >> $1 /(d \omega)$ not only at the first resonance but also for a wide range of values of $g$. An enhanced response amplitude at the low-frequency $(\omega)$ can be achieved by applying two high-frequency forces. Interestingly, and in contrast to this result, when the system is driven by the force $F_{l}(t)$, multiple resonance peaks occur for a wide range of values of $g$ and value at the resonance is $Q=1 / d \omega$. But an enhanced response amplitude at the lowfrequency $(\omega)$ can be achieved by applying only one high-frequency force. 


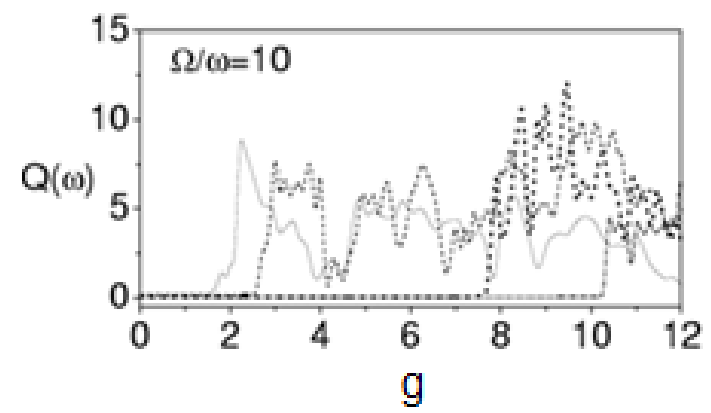

Fig. 2. Response amplitude $Q$ versus the control parameter $g$ for the frequency ratio $\Omega / \omega=10$ and four values of $\beta$ in the system- 1 . The values of $\beta$ for solid, dashed, dotted, dashed, and dot curves are $3,5,15$ and 20 , respectively. The rest of the parameters fixed at $\omega_{0}^{2}=1.0, d=0.5, f=0.1, \omega=$ 0.1 and $\Omega=1.0$.

The system (1) exhibits hysteresis and jump phenomenon when the control parameter $\mathrm{g}$ is varied smoothly from small to a larger and then back to a smaller. As an example, we analyze this phenomenon for $\beta=20$ (seven-well potential (Fig. 1d)). Fig. 4(a) presents $Q$ obtained by varying $g$ in the forward and reverse directions. We can clearly observe hysteresis and a jump phenomenon. Fig. 4(b) shows the magnification of $Q$ curve in the interval $g \epsilon[8,10]$. $Q$ is found to follow different paths as indicated by an arrow when $g$ is varied in the forward and reverse directions. Such behavior of $Q$ is observed in all potential wells except single-well potential.

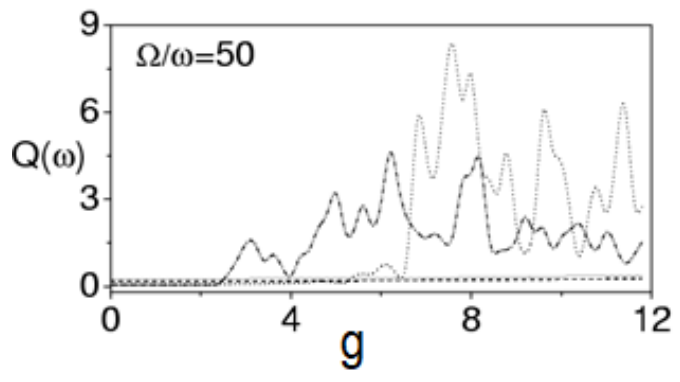

Fig. 3. Response amplitude $Q$ versus the control parameter $g$ for the frequency ratio $\Omega / \omega=50$ and four values of $\beta$ in the system-1. The values of $\beta$ for solid, dashed, dotted, dashed, and dot curves are $3,5,15$ and 20 , respectively. The rest of the parameters fixed at $\omega_{0}^{2}=1.0, \mathrm{~d}=0.5, \mathrm{f}=0.1, \omega=$ 0.1 and $\Omega=5.0$.

We describe the response curve in Fig. 3 for $\beta=15$ (dotted curve). Fig. 5 shows the trajectory plots for a few values of $g$ in the interval $[0,20]$ for $\omega=0.1, \Omega=5.0$ and $\beta=$ 15. For $0<g<10$ the orbits are confined to one well only. There is no cross-well motion (Figs. 5(a) and 5(b)). At $g>10.0$, the orbit lies in one well during the one-half cycle of the drive cycle of the low-frequency signal, and in the other wells during the residual half of the cycle is shown in Figs. 5(c) and 5(d) for $g=15.0$ and $g=20.0$. That is, the particle transmits between the two wells regularly with the period of the low-frequency signal. This is the principle that the weak low-frequency signal is amplified in VR. 

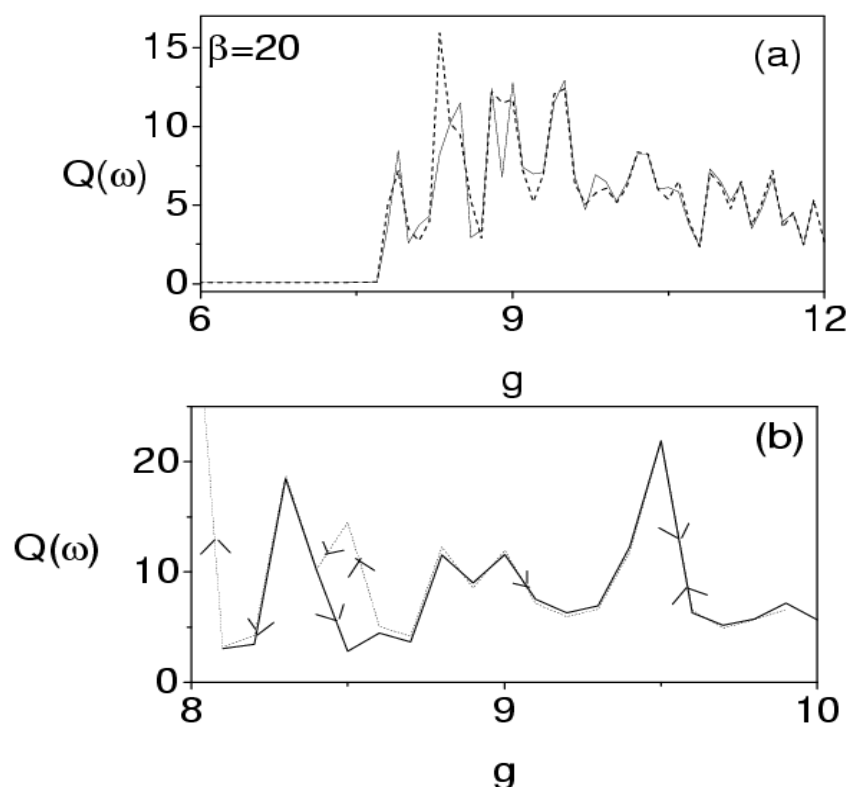

Fig. 4. (a) Response amplitude curves obtained by varying the control parameter $g$ from 6 to 12 (continuous curve) and 12 to 6 (dashed curve) for the seven-well trapped potential system driven by an AM signal. (b) Magnification of the $Q(\omega)$ curves in the interval $g \epsilon[8,10]$ indicating hysteresis and jumps in $Q$. The parameter values of system1 as in Fig. 3.

Next, we analyze the influence of the parameter $\Omega$ on resonance for small and large amplitudes $(g)$ of the AM signal, namely, $g=0.2$ and $g=2.0$ with $\beta=3,5,15,20$. The results are presented in Figs. 6 and 7. The variation of $Q$ versus $\Omega$ is plotted in Fig. 6 for $g$ $=0.2$ and four values of $\beta$, namely, $\beta=3,5,15,20$. For $g=0.2$, we notice only one resonance at $\Omega=0.2$ for all values of $\beta$, but a maximum of the $Q$ decreases with the increase of $\beta$ values, which is clearly evident in Fig. 6 . That is, as the number of potentials well increases, the maximum value of the response amplitude $Q$ decreases. For example, for single-well potential $(\beta=3.0), Q_{\max }=0.6$, for three-well potential $(\beta=5), Q_{\max }=$ 0.3981 , for five-well potential $(\beta=15), Q_{\max }=0.1256$ and for seven-well potential $(\beta=$ 20), $Q_{\max }=0.0984$. For $g=2.0$, the number of resonances decreases with the increase of $\beta$ values, shown in Fig. 7 .

For example, in Fig. 7(a), for $\beta=3$, six resonances occur when $\Omega<2.0$ and no resonances occur for $\Omega>2.0$. For $\beta=5$, single resonance is observed in the interval 0 $<\Omega<0.4678$. No resonances occur in the interval $0.4678<\Omega<1.4258$ and $2.2431<\Omega$ $<5.0$. Two resonances occur in the interval $1.4258<\Omega<2.2431$ which is clearly evident in Fig. 7(b). For $\beta=15$ and 20 only one resonance is observed at $\Omega=0.25$ but $Q_{\max }$ decreases when $\beta$ increases, which is clearly seen in Fig. 7(c) and Fig. 7(d). 

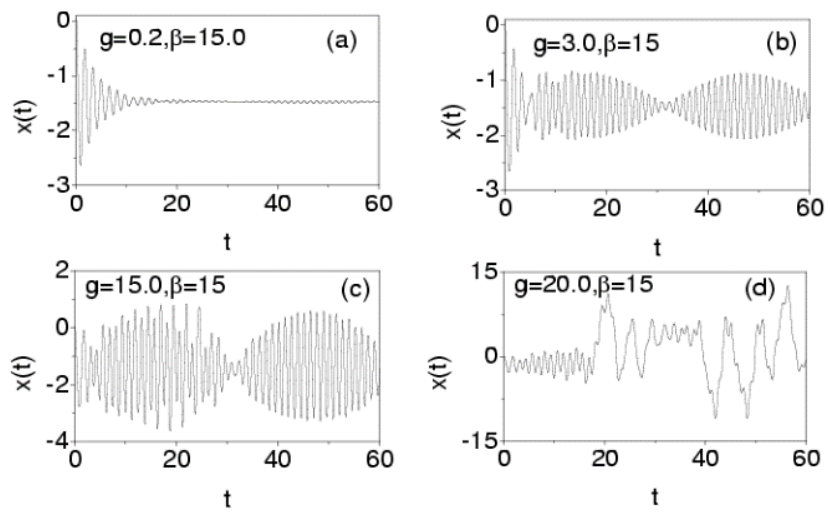

Fig. 5. Trajectory plots for different values of high frequency amplitude $g$ with $\beta=15$ in the system1. The rest of the parameters fixed at $\omega_{0}{ }^{2}=1.0, d=0.5, f=0.1, \omega=0.1$ and $\Omega=5.0$.

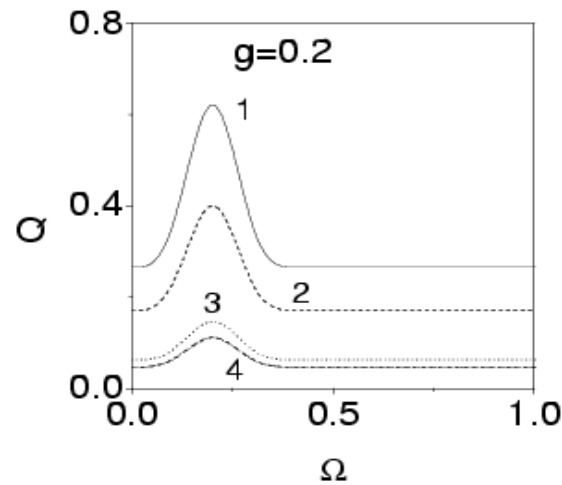

Fig. 6. Response amplitude $Q$ versus the control parameter $\Omega$ for four values of $\beta$ in the system- 1 . The values of $\beta$ for the curves $1,2,3$ and 4 are 3,5,15 and 20 respectively. The rest of the parameters fixed at $\omega_{0}^{2}=1.0, d=0.5, f=0.1, \omega=0.1$ and $g=0.2$.
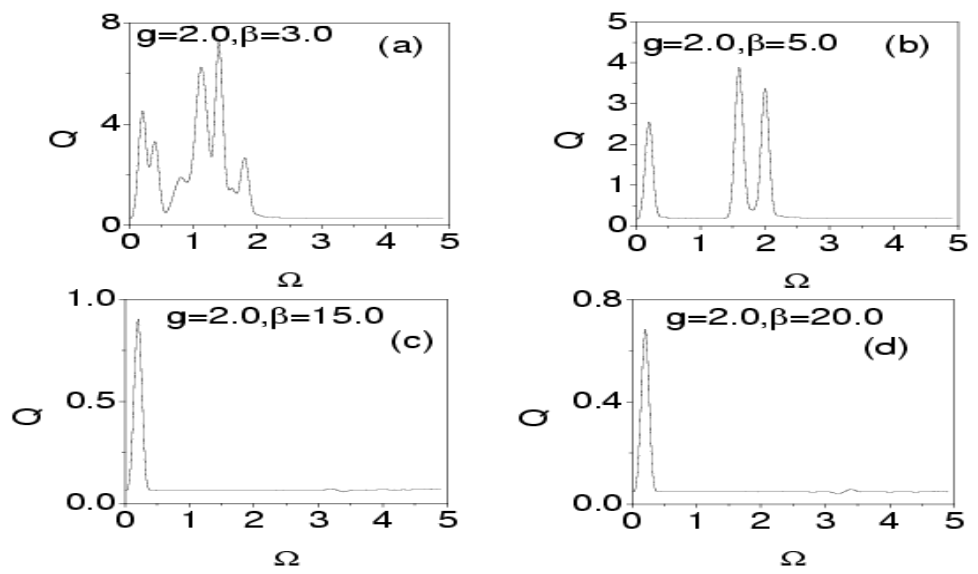

Fig. 7. Response amplitude $Q$ versus the control parameter $\Omega$ for four values of $\beta$ in the system-1. The rest of the parameters fixed at $\omega_{0}^{2}=1.0, d=0.5, f=0.1, \omega=0.1$ and $g=5.0$. 


\subsection{Analysis of response amplitude $Q$ with $F M$ signal}

In this section, we study the effect of FM signal on VR in system-2. We numerically analyze the influence of the parameters $g$ and $\Omega$ on resonance for single-well $(\beta=3)$, triple-well $(\beta=5)$, five-well $(\beta=15)$ and seven-well $(\beta=20)$ potentials in system- 2 . Fig. 8 presents the results. Fig. 8(a) shows the variation of the response amplitude $Q$ with $g$ for single-well $(\beta=3)$, triple-well $(\beta=5)$, five-well $(\beta=15)$ and seven-well $(\beta=20)$ potentials with the frequency's ratio $\Omega / \omega=10$. For all the potential wells, resonance occurs at $g=3.839$, but the corresponding maximum of the $Q$ values decreases while increases the number of potential wells. The variation of the response amplitude $Q$ with $\mathrm{g}$ for frequencies ratio $\Omega / \omega=50$ is reported in Fig. 8(b). For single-well $(\beta=3.0)$ and triplewell $(\beta=5.0)$ potentials, no resonance is observed. Single resonance is observed for fivewell $(\beta=15)$ and seven-well $(\beta=20)$ potentials at $g=3.088$, but $Q_{\max }$ decreases with the increase of a number of potential wells, which is clearly evident in Fig. 8. The dependence of $Q$ on the frequency $(\Omega)$ of the FM signal is studied in Fig. 9 for single-well $(\beta=3)$, triple-well $(\beta=5)$, five-well $(\beta=15)$ and seven-well $(\beta=20)$ potentials in system-2 with $g=0.2$ and $g=2.0$. Fig. 9(a) presents the curves of the response amplitude $Q$ versus the high frequency $\Omega$ of the FM signal for single-well $(\beta=3)$, triple-well $(\beta=5)$, five-well $(\beta$ $=15)$ and seven-well $(\beta=20)$ potentials in system- 2 with $g=0.2, f=0.1$ and $\omega=0.1$. Fig. 9(a) shows that there are only a few $\Omega$ make the response amplitude $Q$ achievable at the maxima for the different potential wells. We call the signal frequency $\Omega$, which makes the response amplitude $Q$ reach its maximum as the optimal high frequency. With the increase of the frequency of the high-frequency signal, the curve in one period (in Fig. 9(a)) turns to double peaks, as is shown in Fig. 9(b) for $g=2.0$. It is similar to the perioddoubling bifurcation that is a bifurcation behavior in the random dynamics. In addition, it can be found that the appearance of the peak is approximated equal to the cycle of the high-frequency signal.
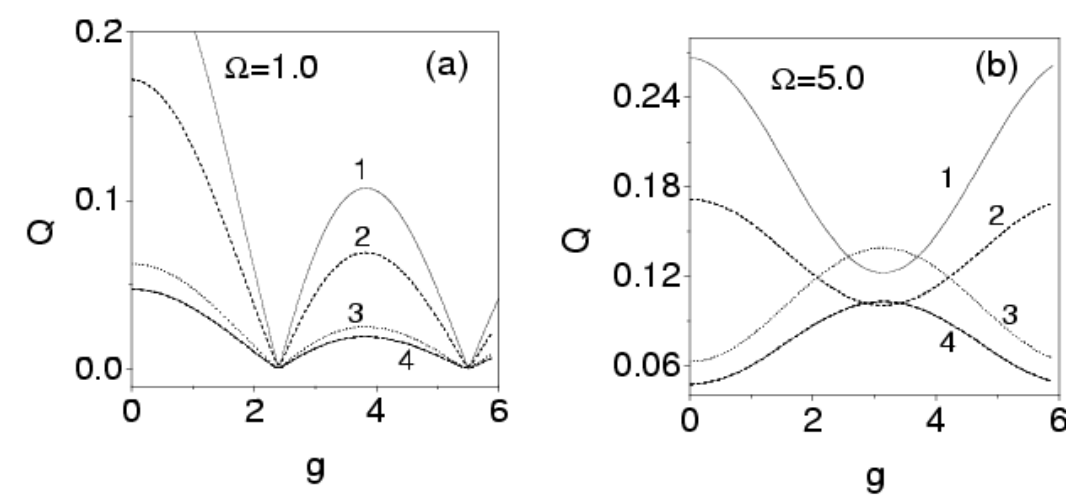

Fig. 8. Response amplitude $Q$ versus the control parameter $g$ for four values of $\beta$ with (a) $\Omega=1.0$ and (b) $\Omega=5.0$ in the system-2. The values of $\beta$ for the curves $1,2,3$ and 4 are $3,5,15$ and 20 respectively. The rest of the parameters fixed at $\omega_{0}^{2}=1.0, d=0.5, f=0.1$ and $\omega=0.1$. 

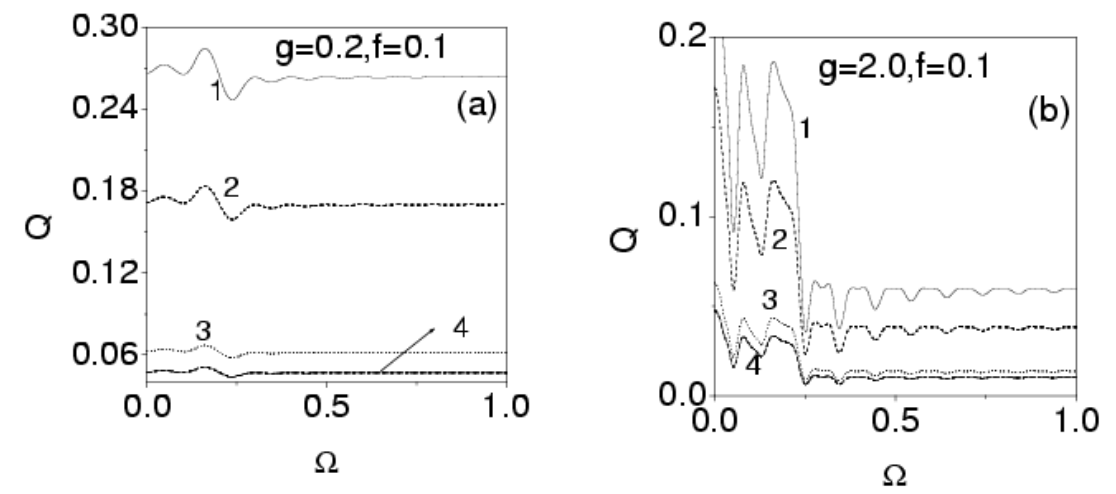

Fig. 9. Response amplitude $Q$ versus the control parameter $\Omega$ for four values of $\beta$ with (a) $g=0.2$ and (b) $g=2.0$ in the system-2. The values of $\beta$ for the curves $1,2,3$ and 4 are $3,5,15$ and 20 respectively. The rest of the parameters fixed at $\omega_{0}^{2}=1.0, d=0.5, f=0.1$ and $\omega=0.1$.

\section{Conclusion}

The effect of modulated signal on the vibrational resonance in a harmonically trapped potential system is numerically studied in this paper. We consider two forms of modulated signals such as amplitude modulated signal and frequency-modulated signals. Depending upon the parameters $\omega_{0}{ }^{2}$ and $\beta, V(x)$ admit an odd number of potential wells. We numerically analyzed the effect of modulated signals on vibrational resonance in systems (1-2) for single-well, three-well, five-well, and seven-well potentials. The influence of the parameter such as $g$ and $\Omega$ on these potential wells is studied. Based on numerical analysis, single resonance and multiple resonances are observed in certain parametric ranges. Due to modulated signals, considerable enhancement in the weak periodic signal occurs in all the potential wells. Hysteresis and a jump phenomenon is observed in the system-1, but such behavior is not observed in system-2. We believe that the results reported in these systems will lead to the detailed analysis of other types of resonance such as stochastic, coherence, and auto resonance in the systems (1-2), resulting in the further understanding of these resonances and their applications.

\section{References}

1. K. Joshi, I. Tiwari, A. Nandi, and P. Parmananda, Phys. Rev. E 98, ID 012218 (2018). https://doi.org/10.1103/PhysRevE.98.012218

2. G. Zhang, D. Hu, and T. Zhang, Access IEEE 7, 58435 (2019). https://doi.org/10.1109/ACCESS.2019.2950692

3. E. Mompo, M. Ruiz-Garcia, M. Carretero, H.T. Grahn, Y. Zhang, and L.L. Bonilla Phys. Rev. Lett. 121, ID 086805 (2018). https://doi.org/10.1103/PhysRevLett.121.086805

4. M. E. Yamakou and J. Jost, Phys. Rev. E 100, ID 022313 (2019). https://doi.org/10.1103/PhysRevE.100.022313

5. A. Kovaleva, Philos. Trans. R. Soc. A 375, 20160213 (2017). https://doi.org/10.1098/rsta.2016.0213

6. O. A. Sultanov, Ufa Mathematical J. 7, 58 (2015). https://doi.org/10.13108/2015-7-1-58 
7. S. Rajamani and S. Rajasekar, arXiv:1510.07887v1 [nlin.CD], 27 Oct 2015.

8. S. Rajamani, S. Rajasekar, and M. A. F. Sanjun, Communicat. Nonlinear Sci. Numer. Simulat. 19, 4003 (2014). https://doi.org/10.1016/j.cnsns.2014.04.006

9. K. Abirami, S. Rajasekar, and M. A. F. Sanjuan, Int. J. Bifur. Chaos 24, 1430031 (2014). https://doi.org/10.1142/S0218127414300316

10. K. Robert and F. Walter, Inter. J. Bifur. Chaos 11, 1607 (2001). https://doi.org/10.1142/S0218127401002870

11. S. Nobukawa, H. Nishimura, and T. Yamanishi, Scientific Reports 7, 1 (2017). https://doi.org/10.1038/s41598-017-01511-y

12. K. S. Oyeleke, O. I. Olusola, U. E. Vincent, D. Ghoshd, and P. V. E. McClintock, Phys. Lett. A 387, ID 127040 (2021). https://doi.org/10.1016/j.physleta.2020.127040

13. S. Surappa and F. L. Degertekin, J. Acoust. Soc. Am. 145, 1881 (2019). https://doi.org/10.1121/1.5101815

14. P. S. Landa and P. V. E. McClintock, J. Phys. Math. Gen. 33, L433 (2000). https://doi.org/10.1088/0305-4470/33/45/103

15. M. Gitterman, J. Phys. A 34, L355 (2001). https://doi.org/10.1088/0305-4470/34/24/101

16. J. Zhu, C. Kong, and X. Liu, Phys. Rev. E 94, ID 032208 (2016). https://doi.org/10.1103/PhysRevE.94.032208

17. Y. Pan, F. Duan, F. Chapeau-Blondeau, L. Xu, and D. Abbott. Philos. Trans. R. Soc. A 379. ID 20200235 (2021). https://doi.org/10.1098/rsta.2020.0235

18. S. Guruparan, V. Ravichandran, S. Selvaraj, V. Chinnathambi, and S. Rajasekar, Ukraine J. Phys. 62, 51 (2017). https://doi.org/10.15407/ujpe62.01.0051

19. I. I. Blckhman and P. S. Landa, Int. J. Non-Linear Mech. 39, 421 (2004). https://doi.org/10.1016/S0020-7462(02)00201-9

20. J. P. Baltanas, L. Lopez, I. Blechman, P. S. Landa, A. Zaikin, and J. Kurths, Phys. Rev. E 67, ID 066119 (2003). https://doi.org/10.1103/PhysRevE.67.066119

21. V. N. Chizhevsky, E. Smeu, and G. Giacomelli, Phys. Rev. Lett. 91, 220602 (2003). https://doi.org/10.1103/PhysRevLett.91.220602

22. V. N. Chizhevsky and G. Giacomelli, Phys. Rev. E 73, ID 022103 (2006). https://doi.org/10.1103/PhysRevE.73.022103

23. S. Jeyakumari, V. Chinnathambi, S. Rajasekar, and M. A. F. Sanjuan, Phys. Rev. E, 80, ID 046608 (2009). https://doi.org/10.1103/PhysRevE.80.046608

24. S. Jeyakumari, V. Chinnathambi, S. Rajasekar, and M. A. F. Sanjuan, Int. J. Bifur. Chaos 21, 275 (2011). https://doi.org/10.1142/S0218127411028416

25. J. H. Yang and X. B. Liu, arXiv: 1106.3431 [astro-ph], (2001).

26. K. Abirami, S. Rajasekar, and M. A. F. Sanjuan, Chaos 21, ID 033106 (2011). https://doi.org/10.1063/1.3610213

27. A. DazaAlvar, D. Wagemakers, S. Rajasekar, and M. A. F. Sanjun, Commun. Nonlinear Sci. Numer. Simul. 18, 411 (2013). http://dx.doi.org/10.1016/j.cnsns.2012.07.010

28. V. Ravichandran, V. Chinnathambi, and S. Rajasekar, Physica A 376, 223 (2007). https://doi.org/10.1016/j.physa.2006.11.003

29. L. Ravisankar, V. Ravichandran, V. Chinnathambi, and S. Rajasekar, Chin. J. Phys. 52, 1026 (2014).

30. S. Guruparan, B. R. D Naayagam, V. Ravichandran, V. Chinnathambi, and S. Rajasekar, Chem. Sci. Rev. Letts. 49, 870 (2015)

31. M. V. S. Meenakshi, S. Athisayanathan, V. Chinnathambi, and S. Rajasekar, Chinese J. Phys. 55, 2208 (2017). https://doi.org/10.1016/j.cjph.2017.09.009

32. V. B. S. Jothi, S. Selvaraj, V. Chinnathambi, and S. Rajasekar, Annual Rev. Chaos Theory, Bifurcations Dynamical Syst. 7, 56 (2017).

33. K. Abirami, S. Rajasekar, and M. A. F. Sanjuan, Commun. Nonlinear Sci. Numer. Simulat. 47, 370 (2017). https://doi.org/10.1016/j.cnsns.2016.12.002

34. Z. Yang and L. Ning, Pramana. J. Phys. 89, 1 (2019). https://doi.org/10.1007/s12043-019-1750-2 